\title{
COMMUNICATION
}

\section{Proposition d'un protocole de prise en charge implanto-portée de patients traités en oncologie tête et cou suite à une étude rétrospective au CHRU de Brest}

\author{
E. Hascoet (Brest), G. Valette (Brest), G. Le Toux (Brest), S. Boisramé (Brest)
}

L'organisation de la réhabilitation maxillo-faciale implanto-portée en oncologie tête et cou reste sujet à débat. Il n'existe pas de consensus quant au moment de la réhabilitation implantaire (pendant ou à distance de l'exérèse) ou quant aux prérequis anatomiques qui garantissent son succès. Le but de cette étude est de mettre en place un protocole de prise en charge implanto-portée de patients ayant été traités en oncologie tête et cou. Il s'agit d'une étude rétrospective entre 2013 et 2017, analysant la réhabilitation implantoportée de neuf patients traités pour un cancer tête et cou par chirurgie et radiothérapie (groupe $1: 5$ patients) ou par chirurgie seule (groupe $2: 4$ patients). Les critères étudiés étaient les données personnelles des patients, leurs antécédents oncologiques, les bilans oraux pré-opératoires effectués, les thérapeutiques utilisées ainsi que le suivi. Tous les patients ont été traités pour des carcinomes épidermoïdes majoritairement situés au niveau du plancher buccal. Ils présentaient tous une limitation de la mobilité linguale, une absence de vestibule et des tissus crestaux mobiles. Les projets prothétiques prévoyaient de réaliser pour sept patients une prothèse amovible complète mandibulaire stabilisée sur implants et pour deux patients des couronnes unitaires sur implant. Les patients ayant reçu un lambeau libre de péroné latéral ont bénéficié d'une greffe d'apposition associée à un lambeau de FAMM. Les deux greffes ont permises la pose des implants. Sur neuf patients, cinq ont bénéficié d'une libération linguale, six ont reçu une vestibuloplastie et huit ont eu une greffe épithélio-conjonctive péri-implantaire. Les implants ont été posés sous anesthésie générale avec un guide chirurgical pointeur. Il s'agissait d'implants Tissu Level RN SLA Roxolid (Straumann, Basel, Suisse). Pour les patients du groupe 2, la pose des implants a eu lieu en même temps que la vestibuloplastie. Lors de cette étude quatre patients étaient réhabilités par une prothèse sur implants. Le groupe 2 n'a pas présenté de complications. Deux patients du groupe 1 ont nécessité plusieurs libérations linguales et deux implants ne se sont pas ostéointégrés dans ce groupe. Lors de chirurgies en deux temps, des greffes épithélio-conjonctives crestales ont dû être réalisées pendant la vestibuloplastie puis pendant la pose implantaire. Deux greffes osseuses ont été réalisées chez deux patients ayant été irradiés bien que cela soit contre indiqué dans la littérature (Raoul 2009). Une greffe osseuse d'apposition peut donc être une solution viable chez ces patients afin de limiter le bras de levier prothétique. Cette étude a permis de mettre en exergue : l'importance de prévoir en amont la réhabilitation implantoportée lors de la reconstruction pour une optimisation du positionnement du greffon osseux et l'intérêt d'effectuer dans le même temps vestibuloplastie, pose des implants et aménagement péri-implantaire pour la pérennité implantaire. Cette étude rétrospective a permis de proposer un protocole de prise en charge standardisé de la réhabilitation implanto-portée de patients traités pour un cancer tête et cou. 